\title{
Intercellular Communication Process
}

National Cancer Institute

\section{Source}

National Cancer Institute. Intercellular Communication Process. NCI Thesaurus. Code C16395.

Any process by which signals are passed from one cell to another through a direct multicellular interaction. This process is involved widely in communication, and is seen in immune response, neuronal transmission, inflammation and other processes. 